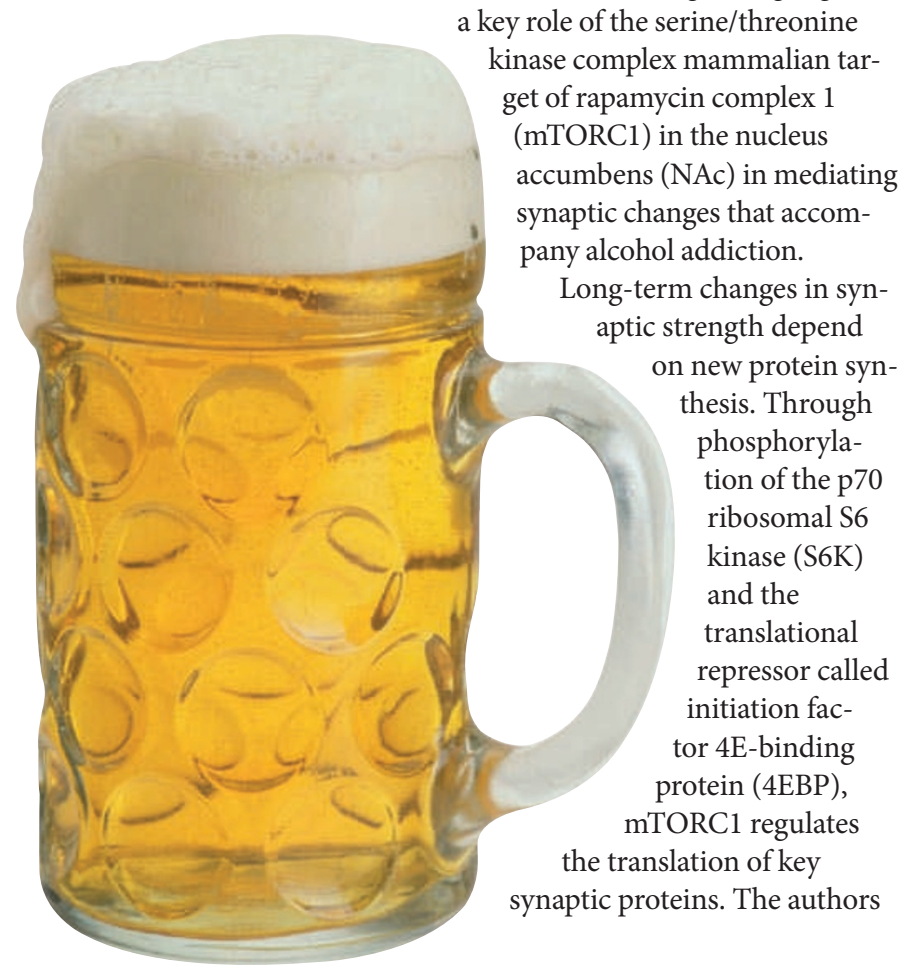

\title{
ADDICTION
}

\section{A sobering thought}

Drugs of abuse, including alcohol, can promote maladaptive synaptic plasticity mechanisms in the brain's reward system, but the underlying molecular pathways are not well characterized. Now, Ron and colleagues highlight

key role of the serine/threonine

kinase complex mammalian taraccumbens (NAc) in mediating synaptic changes that accomany alcohol addiction.

g-term changes in synic strength depend new protein synhesis. Through phosphorylation of the p70 ribosomal S6 kinase (S6K) and the translational repressor called initiation factor $4 \mathrm{E}$-binding protein (4EBP), mTORC1 regulates the translation of key roteins. The authors therefore sought to characterize mTORC1 activity in alcohol addiction, and focused on the NAc as previous studies had shown druginduced plasticity in this region.

The authors showed that mTORC1 activity, as measured by phosphorylation of S6K and 4EBP, was enhanced in the NAc of rodents following excessive alcohol consumption, and remained so for 24 hours after alcohol withdrawal. Next, they studied the effects of the mTORC1 inhibitor rapamycin on alcoholrelated behaviours. Rapamycin reduced conditioned place preference in response to alcohol, indicating decreased reward-seeking behaviour. It also decreased locomotor sensitization - a phenomenon in which repeated exposure to alcohol results in enhanced responsiveness of mice to successive doses.

To create a binge-drinking paradigm, mice and rats were trained to drink large quantities of alcohol at repeated intervals. A single systemic dose of rapamycin 3 hours before a drinking session decreased the motivation to binge drink and excessive consumption. By contrast, water and sucrose consumption remained unchanged, demonstrating that the effects of rapamycin were alcohol specific.

At the molecular level, excessive alcohol consumption was associated with increased expression of the scaffolding protein homer and the GluA1 subunit of the AMPA ( $\alpha$-amino3-hydroxy-5-methyl-4-isoxazole propionic acid) receptor in the NAc. Both proteins are known synaptic targets of mTORC1 and have been linked to drug-induced plasticity in this region.

These studies suggest that mTORC1-dependent alterations in synaptic protein expression might regulate the long-term changes underlying alcohol-related disorders, highlighting mTORC1 as a potential therapeutic target in treating addiction.

Safia Ali Danovi

ORIGINAL RESEARCH PAPER Neasta, J. et al.

Role for mammalian target of rapamycin complex 1 signaling in neuroadaptations underlying alcohol-related disorders. Proc. Natl Acad. Sci. USA 107, 20093-20098 (2010) 